\title{
Overconfident Newsvendor's Joint Pricing and Ordering Decisions under the Additive Demand Case
}

\author{
Li Ying $^{1} \&$ Guo Gui-hang ${ }^{1}$ \\ ${ }^{1}$ School of English for International Business, Guangdong University of Foreign Studies, China \\ Correspondence: Guo Gui-hang, School of English for International Business, Guangdong University of Foreign \\ Studies, No.2 Baiyundadaobei, Guangzhou 510420, P.R.China. E-mail: guihang@gdufs.edu.cn
}

Received: June 4, 2018

doi:10.5539/ijbm.v13n11p135

\author{
Accepted: July 20, 2018 \\ Online Published: October 12, 2018 \\ URL: https://doi.org/10.5539/ijbm.v13n11p135
}

\begin{abstract}
Booming cross-border e-commerce in China has made market demand become more uncertain and volatile, which has imposed more challenges on the pricing and ordering decisions of retailers. More behavioral and cognitive factors need to be taken consideration in operations management and inventory management in particular. Aiming at newsvendor's overconfidence psychology, we define newsvendor overconfidence under the additive demand case where demand is price dependent, construct the decision model, derive the optimal joint pricing and ordering decisions and analyze structural properties. The analysis proposes the sufficient condition for the existence of the unique optimal price and order quantity. And we find that the optimal price and stock factor both increase with newsvendor's overconfidence level. Finally, the effect of overconfidence level on optimal decisions is verified through a numerical example and some practical implications are highlighted.
\end{abstract}

Keywords: additive demand, overconfident newsvendor, price, order

\section{Introduction}

With the improved accessibility of Internet and widespread use of cross-border e-commerce platforms, more and more transactions are completed online. In 2017, the cross-border e-business volume in China reached RMB3,100 billion yuan. It is estimated that up to 2020, the size of China's cross-border e-business will amount to 12,000 billion yuan, accounting for about $37.6 \%$ of the total volume of China's imports and exports. While online purchase has facilitated the convenience to consumers, it also makes it more difficult for retailers to forecast market demand because consumers have more alternatives in choosing sellers. Since inventory is one of most expensive assets of many companies, an effective inventory management is becoming more and more crucial for sellers facing uncertain and volatile market demand. While excessive inventory will take up financial and human resources of business enterprises, insufficient inventory level may result in failures to meet market demand promptly in the situations of changing demand, thus loosing the opportunity of making a higher profit.

As the foundation of various inventory management models, newsvendor model is widely applied in the production management of short-life-cycle products with uncertain demand as well as in the ordering practice of retailing industry. Traditional researches on newsvendor model are based on the hypothesis of "rational economic man" and the assumption that decision-makers have stable preferences and ability of making rational decisions. That is, decision-makers are totally rational and can anticipate the consequences of their decisions without being affected by psychological and behavioral factors. However, more and more researches reveal that the actual decision-making behaviors diverge from the findings of previous theoretical researches. Based on their experimental research, Kahneman and Tversky (1974) prove that decisions are affected by psychological perceptions and that decision-makers are not totally rational, but boundedly rational. Decision-makers' behavioral factors will influence their decisions. For example, in such operational decisions as ordering decisions, the optimal solution of actual order quantity of newsvendors who are affected by such behavioral factors as anchoring and learning factors has systematic deviation from that of the newsvendors who are totally rational (Schweitzer \& Cachong,2000; Bolton \& Katok, 2008). Therefore, the behavioral and cognitive factors of decision makers should be taken into consideration when making operational decisions (Gino \& Pisano, 2008).

Empirical researches have shown that behavioral factors like overconfidence exist in the decision-making process in uncertain situations. In making pricing and ordering decisions, some retailers know about the market demand clearly, while others might have misperception about the market demand with overconfidence. Although 
many studies have considered the joint decision problem where the price and order quantity are both decision variables, there has limited work taking overconfidence into consideration. In this paper, we consider the stockout penalty cost and study overconfident newsvendor's joint pricing and ordering problem when demand is price dependent. We try to understand: (1) what is the overconfident newsvendor's optimal price and ordering quantity? (2) how does newsvendor's overconfidence affects his optimal price and ordering quantity? Hopefully, our study may provide some guidance for retailers on how to set price and ordering quantity in the current booming e-commerce business context in China where market demand is becoming uncertain and volatile.

\section{Literature Review}

Newsvendor problem, as the foundation in inventory management, is widely used in short-life-cycle products' production management. The traditional newsvendor model assumes that the price is given and not decided by the newsvendor. Whitin (1955) extended this model by setting price as a decision variable. He combined the inventory control and price theory within economic theory and used aspects of the demand function of economic theory to develop inventory control theory. Based on his findings, he suggested that, while economists and businessmen aim to realize profit maximization, they should also consider realistic methods of cost minimization. Since then, many scholars have studied newsvendor' $\mathrm{s}$ joint pricing and ordering decisions under different demand cases. For example, Petruzzi and Dada (1999) reviewed the existing research on joint pricing and ordering decisions. They suggested that incorporating market parameters such as demand and selling price into the model could provide an excellent vehicle for examining how operational problems interact with marketing issues to influence decision making at the firm level. In their research, they extended the newsvendor problem by setting stocking quantity and selling price simultaneously. Li et al. (2005) considered the impact of the coordination between manufacturer and retailer, and demand uncertainty on firm's pricing order decision. In their research, they find that the extent of influence of increasing demand variability on the decisions depends on the type of demand uncertainty faced. In particular, for additive demand uncertainty, both price and service level decrease in demand variability. Zhang et al. (2008) studied the joint decision on pricing and inventory control in a single period. Their research proves the existence and uniqueness of the optimal policy in the general demand function. They also prove that the optimal price is decreasing with the inventory level and that the optimal order quantity is also decreasing with the price. Liu et al. (2013) studied the newsvendor's joint pricing and ordering problem with shortage penalty under additive and multiplicative demand cases. Their research proved the effect of price sensitivity of demand on the optimal decisions and revenue and might serve as the modeling framework for other related research such as multi-retailer competition and supply chain contract coordination.

Some scholars have conducted behavioral experiments in the study of newsvendor problem. Their experimental evidences confirm that decision-makers' behavioral factors affect their decision making, which is different from the rational decision-making assumption made in above analysis. For example, Schweitzer \& Cachon (2000) studied the decision bias in the newsvendor problem with a known demand distribution. Their experiments examine newsvendor decisions across different profit conditions and demonstrate that choices systematically deviate from those that maximize expected profit. Based on their experimental results, they conclude that a better understanding of actual behavior in real processes may suggest the modifications of traditional assumptions and that new techniques need to be developed to correctly optimize these systems. Bolton \& Katok (2008) studied learning-by-doing in the newsvendor problem through a laboratory investigation. They find that the actual order quantity of the newsvendor, who is affected by behavioral factors such as anchoring and learning, has a systematic deviation from rational newsvendor's optimal decision. Through experiment, they identify several institutional factors that may facilitate optimal stocking. Bolton and Katok suggest that it may be critical to keeping people from over-reacting to short-term fluctuations by inhibiting inappropriate responses to short-term information and that knowledge gained through personal experience may help to achieve remarkable improvement in performance.

Scholars suggest that research in operations management need to take behavioral and cognitive factors into consideration, such as different aspects of individual decision bias: loss aversion, hyperbolic time preference, and so on. Özer and Zheng (2011) analyzed the effects of behaviors on pricing management from three aspects including individual choice theory, social preference and bounded rationality. Loch and Wu (2007) analyzed in detail four aspects of individual decision bias including reference dependence and loss aversion, hyperbolic time preference, regret theory, heuristics and bias and their application in the operations management. Zhang et al (2015) studied the joint ordering and pricing problem of the loss-averse retailer. They conclude that the loss-aversion behavior has an effect on optimal decisions and expected utility and that the retailer tends to take conservative quantity policies that make order quantity closed to mean demand due to his/her loss-aversion behavior. Chen et al (2016) considered manufacturer's joint ordering and pricing in a supply chain composed of 
rational manufacturer and overconfident sales agent. Overconfidence is a typical cognitive bias in individual decision-making, which includes overestimation in one's actual performance, overplacement in one's performance relative to others, and excessive precision in one's beliefs (Moore \& Healy, 2008).

Croson et al. (2008) put forward a theoretical model of overconfident newsvendor, verified that overconfidence lead to suboptimal decision-making with mathematical analysis. Ren et al. (2013) used behavioral experiment to show that, underestimation of the market demand variance lead to decision deviation from the optimal order decision, provided support for the hypothesis that newsvendor's overconfidence was one reason for the suboptimal order decision. These studies did not consider the joint decision problem, where the price and order quantity are both decision variables. Zhang (2016) assumed no constrains on manufacturer's production capacity, studied the impact of overconfidence on retailer's joint pricing and ordering when demand is uncertain and price dependent. However, in reality, the retailer might face the stockout and result in the penalty cost.

In view of this, under the additive demand case, this paper considers the stockout penalty cost, studies overconfident newsvendor's joint pricing and ordering problem when demand is price dependent. With consideration of price and penalty cost, the sufficient condition for the existence of the unique optimal price and order quantity is obtained. The effect of the overconfidence on the optimal decision is analyzed, and verified through a numerical example.

\section{Model Setting}

\subsection{Newsvendor Overconfidence Setting When Demand Is Price Dependent}

There is one newsvendor selling products over one selling period. Let $p$ represent the selling price. Under additive demand case, the actual market demand is: $D(p, \epsilon)=y(p)+\epsilon$. It is composed of a price dependent mean demand $y(p)=a-b p(a>0, b>0)$ and a random variable $\epsilon$ defined on range $[A, B]$, with the mean $\mu$, the variance $\sigma^{2}$, the probability density function $f(\cdot)$, the cumulative distribution function $F(\cdot)$, and the failure rate function $h(\cdot)=f(\cdot) /[1-F(\cdot)] . \epsilon$ is set to have the increasing failure rate (IFR), which is shared by common distributions, such as normal, uniform, and weibull distributions.

Using the overconfidence modeling in Croson et al.(2008), we assume the price dependent demand in overconfident newsvendor's belief $D_{O}(p, \epsilon)$ has the following representation form with the actual market demand $D(p, \epsilon)$ :

$$
D_{0}(p, \epsilon)=\gamma D(p, \epsilon)+(1-\gamma) E(D(p, \epsilon))=y(p)+\gamma \epsilon+(1-\gamma) \mu
$$

where $(1-\gamma)$ is the overconfidence level, $0 \leq \gamma \leq 1$. When $1-\gamma=0$, the newsvendor is rational.

It is easy to verify that $E\left(D_{O}(p, \epsilon)\right)=E(D(p, \epsilon))=y(p)+\mu, \operatorname{Var}\left(D_{O}(p, \epsilon)\right)=\gamma^{2} \operatorname{Var}(D(p, \epsilon))=\gamma^{2} \sigma^{2}$. This implies that, when demand is price dependent, the means of the demand in overconfident newsvendor's mind is the same as that of the actual market demand, while the demand variance in his mind is less than or equal to that of actual demand.

\subsection{Overconfident Newsvendor's Profit Function}

Let $q$ represent the order quantity, $c$ represent the per-unit order cost, $h$ represent the per-unit disposal cost at the end of the selling period ( $h$ might be negative, but $h \geq-c$ ), $s$ represent the per-unit penalty cost for the stockout.

At the beginning of the selling period, the newsvendor should decide the unit retail price and order quantity to maximize his own expected profit. When the demand is price dependent, the profit function of overconfident newsvendor is:

$$
\Pi(q, p)=\left\{\begin{array}{cc}
p D_{O}(p, \epsilon)-c q-h\left[q-D_{o}(p, \epsilon)\right], & D_{o}(p, \epsilon) \leq q \\
p q-c q-s\left[D_{O}(p, \epsilon)-q\right], & D_{O}(p, \epsilon)>q .
\end{array}\right.
$$

Substituting (1) into above function, we get:

$$
\Pi(q, p)=\left\{\begin{array}{cc}
p[y(p)+\gamma \epsilon+(1-\gamma) \mu]-c q-h[q-[y(p)+\gamma \epsilon+(1-\gamma) \mu]], & D_{O}(p, \epsilon) \leq q, \\
p q-c q-s[[y(p)+\gamma \epsilon+(1-\gamma) \mu]-q], & D_{O}(p, \epsilon)>q .
\end{array}\right.
$$

Let $\frac{q-y(p)-(1-\gamma) \mu}{\gamma}=z$, then profit function could be represented as the function of $z$ and $p$ : 


$$
\Pi(z, p)=\left\{\begin{array}{c}
p[y(p)+\gamma \epsilon+(1-\gamma) \mu]-c[y(p)+\gamma z+(1-\gamma) \mu]-h \gamma(z-\epsilon), \epsilon \leq z, \\
p[y(p)+\gamma z+(1-\gamma) \mu]-c[y(p)+\gamma z+(1-\gamma) \mu]-s \gamma(\epsilon-z), \quad \epsilon>z .
\end{array}\right.
$$

According to the definition of stocking factor in Petruzzi et al.(1999), we get

$$
\mu+\frac{q-E\left(D_{O}(p, \epsilon)\right)}{\sqrt{\operatorname{Var}\left(D_{O}(p, \epsilon)\right)}} \sigma=\mu+\frac{q-y(p)-\mu}{\sqrt{\gamma^{2} \sigma^{2}}} \sigma=z .
$$

This implies that $z$ is actually the stocking factor under additive demand case.

Therefore, when demand is price dependent, the expected profit function of overconfident newsvendor is:

$$
\begin{aligned}
E[\Pi(z, p)]=\int_{A}^{z}\{p[y(p)+\gamma u+(1-\gamma) \mu]-h \gamma(z-u)\} f(u) d u \\
\quad+\int_{z}^{B}\{p[y(p)+\gamma z+(1-\gamma) \mu]-s \gamma(u-z)\} f(u) d u-c[y(p)+\gamma z+(1-\gamma) \mu]
\end{aligned}
$$

Let $\Lambda(z)=\int_{A}^{z}(z-u) f(u) d u, \Theta(z)=\int_{z}^{B}(u-z) f(u) d u$

Then we get:

$$
E[\Pi(z, p)]=(p-c)(y(p)+\mu)-\gamma(c+h) \Lambda(z)-\gamma(p+s-c) \Theta(z)
$$

\section{Optimal Analysis}

Overconfident newsvendor need to decide the optimal ordering quantity $q^{*}$ and retail price $p^{*}$ to optimize the expected profit, which equals to decide the optimal stocking factor and retail price: $\max _{z, p} E[\Pi(z, p)]$.

THEOREM 1. Given $\epsilon$ has the increasing failure rate(IFR), if $a+2 b s-b c+(1-\gamma) \mu+\gamma A>0$, then the optimal order quantity for the overconfident newsvendor is $q^{*}=\gamma z+y\left(p^{*}\right)+(1-\gamma) \mu$, the optimal retail price is $p^{*}=p^{0}-\gamma \Theta(z) / 2 b$, where $p^{0}=(a+b c+\mu) / 2 b . z$ is determined uniquely by $\bar{F}(z)=$ $(c+h) /\left(p^{*}+s+h\right)$.

Proof.

Given $z$, the first and second derivatives of $E[\Pi(z, p)]$ taken with respect to $p$ :

$$
\begin{gathered}
\frac{\partial E[\Pi(z, p)]}{\partial p}=2 b\left(p^{0}-p\right)-\gamma \Theta(z) \\
\frac{\partial^{2} E[\Pi(z, p)]}{\partial p^{2}}=-2 b<0
\end{gathered}
$$

Therefore, the optimal price is determined uniquely as a function of $z$ :

$$
p^{*}=p^{0}-\gamma \Theta(z) / 2 b .
$$

Substituting $p^{*}$ into $E[\Pi(z, p)]$ :

$$
E\left[\Pi\left(z, p^{*}\right)\right]=\left(p^{*}-c\right)\left(y\left(p^{*}\right)+\mu\right)-\gamma(c+h) \Lambda(z)-\gamma\left(p^{*}+s-c\right) \Theta(z)
$$

Let $p(z)$ represent the optimal price $p^{*}$ given $z$ :

$$
\frac{d E[\Pi(z, p(z))]}{d z}=\frac{\partial E[\Pi(z, p(z))]}{\partial z}=\frac{\gamma}{2 b} g(z)
$$

where $g(z)=(a+b c+\mu-\gamma \Theta(z))(1-F(z))+2 b(s-c)-2 b F(z)(h+s)$. Since $\frac{\gamma}{2 b}>0$, there is $z$ which satisfies $g(z)=0$, which can be transformed to $\bar{F}(z)=(c+h) /\left(p^{*}+s+h\right)$, and rewritten as $F(z)=\frac{a-b c+2 b s+\mu-\gamma \Theta(z)}{a+b c+2 b h+2 b s+\mu-\gamma \Theta(z)}$. This shows the existence of $z$. 
$g(z)$ is continuous on range $[A, B], g(A)=a+2 b s-b c+(1-\gamma) \mu+\gamma A, \quad g(B)=-2 b(c+h)<$ $0, g^{\prime}(z)=(1-F(z))\{\gamma(1-F(z))-h(z)(a+b c+\mu-\gamma \Theta(z)+2 b h+2 b s)\}, \quad g^{\prime \prime}(z)=-h(z) g^{\prime}(z)+$ $(1-F(z))\left\{-\gamma f(z)-h^{\prime}(z)(a+b c+\mu-\gamma \Theta(z)+2 b h+2 b s)-h(z) \gamma(1-F(z))\right\}$. If $g(A)>0$, then $g(z)$ has at least one zero point on range $[A, B]$. Given the IFR of $\epsilon$, then $h^{\prime}(z)>0$. When $g^{\prime}(z)=0$, we get $g^{\prime \prime}(z)<0$. Thus, $g(z)$ is unimodal function on range $[A, B]$, which guarantees the uniqueness of $z$.

Theorem 1 gives out the optimal price and stocking quantity for the overconfident newsvendor when demand is price dependent. According to this theorem, we get the properties of stocking factor $z$ and optimal price $p^{*}$ :

COROLLARY 1 The optimal solution has the following structure:

(1) $z \uparrow(1-\gamma)$

(2) $p^{*} \uparrow(1-\gamma)$

Proof.

(1) According to theorem 1,

$$
\bar{F}(z)=\frac{c+h}{p^{0}-\gamma \Theta(z) / 2 b+s+h}
$$

Take first derivative with respect to $\gamma$ :

$$
\frac{d z}{d \gamma}\left\{-\frac{1}{\gamma} h(z)\left(p^{0}-\gamma \Theta(z) / 2 b+s+h\right)+\frac{\bar{F}(z)}{2 b}\right\}=\frac{1}{2 b \gamma} \Theta(z)
$$

Let $p(z)$ represents the optimal price given $z$, we get:

$$
\begin{gathered}
\frac{d E[\Pi(z, p(z))]}{d z}=\gamma\left[\left(p^{0}-\gamma \Theta(z) / 2 b+s+h\right) \bar{F}(z)-(c+h)\right] \\
\frac{d^{2} E[\Pi(z, p(z))]}{d z^{2}}=\gamma^{2} \bar{F}(z)\left[\frac{\bar{F}(z)}{2 b}-\frac{1}{\gamma} h(z)\left(p^{0}-\gamma \Theta(z) / 2 b+s+h\right)\right]
\end{gathered}
$$

Since $z$ maximizes $E[\Pi(z, p(z))]$, we get:

$$
\frac{\bar{F}(z)}{2 b}-\frac{1}{\gamma} h(z)\left(p^{0}-\gamma \Theta(z) / 2 b+s+h\right) \leq 0
$$

Thus $\frac{d z}{d \gamma} \leq 0$.

(2) Since $p^{*}=p^{0}-\gamma \Theta(z) / 2 b$, then $\frac{d p^{*}}{d \gamma} \leq 0$.

Corollary 1 shows that, rational newsvendor's stocking factor and optimal price provide the minimum threshold value for overconfident newsvendor. When the overconfidence level increases, the stocking factor and optimal retail price increase as well, the deviation from the rational newsvendor's optimal decisions become larger.

\section{Numerical Example}

This numerical example is used to illustrate the influence of overconfidence on overconfident newsvendor's stocking factor, optimal retail price, optimal order quantity and expected profit under the additive demand case. $\epsilon$ is set to be the uniform distribution on $[-15,15]$. Other parameters are set to meet the condition $a+2 b s-$ $b c+(1-\gamma) \mu+\gamma A>0: a=40, b=2, c=6, h=-1, s=2$, overconfidence level $(1-\gamma)$ is increased from 0 to 1 in 0.2 increment.

Table 1 Optimal decisions and overconfidence level

\begin{tabular}{ccccc}
\hline$(\mathbf{1}-\boldsymbol{\gamma})$ & $\boldsymbol{Z}$ & $\boldsymbol{p}^{*}$ & $\boldsymbol{q}^{*}$ & $\boldsymbol{E}[\boldsymbol{\Pi}]$ \\
0 & 3.8760 & 12.4844 & 18.9072 & 70.9021 \\
0.2 & 3.9658 & 12.5942 & 17.9842 & 75.9727 \\
0.6 & 4.0513 & 12.7003 & 17.0302 & 81.2308 \\
0.8 & 4.1329 & 12.8032 & 16.0468 & 86.6625 \\
1 & 4.2110 & 12.9030 & 15.0362 & 92.2558 \\
\end{tabular}


As shown in Table 1, stocking factor and optimal price increase with overconfidence level $(1-\gamma)$, the optimal ordering quantity decreases with overconfidence level. This differs from the findings in Croson et al. (2008): overconfident newsvendor will order more product (the low-profit condition) and less product (the high-profit condition) than rational newsvendor. In addition, overconfidence's effect on newsvendor's expected profit shows that: the smaller the variance of the market demand in overconfident newsvendor's mind, in other word, the higher the overconfidence level, the greater the expected profit.

\section{Concluding Remarks}

Many researchers have conducted studies on the newsvendor problem from various perspectives. From the behavioral operation perspective, overconfident newsvendor has a biased belief regarding market demand. When demand is price dependent, the means of the demand in overconfident newsvendor's mind is the same as that of the actual market demand, while the demand variance in his mind is less than or equal to that of actual demand. This paper has defined newsvendor overconfidence when demand is price dependent in single period. We also studied the overconfident newsvendor's joint pricing and ordering problem with consideration of stockout penalty cost. We find that, under the additive demand case, the sufficient condition for the unique existence of the optimal retail price and ordering quantity is given. It is shown that, the stocking factor and the optimal price both increase with overconfident newsvendor's overconfidence level, while the optimal ordering quantity decrease with overconfidence level. This finding is different from the findings of the research conducted by Croson et al (2008) in which they find that overconfident newsvendor will order more product (the low-profit condition) and less product (the high-profit condition) than rational newsvendor.

This research has some practical implications. With the encouraging government policy and the widespread use of cross-border e-commerce platforms, it is expected that the retail volume through cross-border e-commerce in China will increase to account for about $20 \%$ of the total retail business in this country, with an estimated annual growth of $30 \%$. Under this context and with the uncertain and volatile market demand as well as changing consumer preferences, it will become more challenging for decision makers in making pricing and inventory management decisions. Since decision makers will be affected by their overconfidence, it will be more realistic to take this influential factor into consideration when setting decision models. In other words, under the context of uncertain market demand, the results of our research may help the managers of business enterprises to set product prices and ordering quantity in a more effective way so as to realize profit maximization. In addition, we also consider the stockout cost, which may turn out to be more realistic. To some extent, our model achieves a balance between inventory management and the satisfaction of consumer demand. Understanding that overconfidence affects optimal price and ordering quantity, managers may adjust their pricing and ordering decisions accordingly.

There are also limitations of our research. Our model is still simple without taking the overconfidence of consumers into consideration. Also, we did not consider the differences between individual and group overconfidence. Possible extension could be to consider the joint pricing and ordering problem under the multiplicative and general demand function. We expect some of the results in this paper still hold in this extension.

\section{Acknowledgements}

This research was supported with funds from Guangdong University of Foreign Studies (project No.299-X5218113). The support is gratefully acknowledged.

\section{References}

Bolton, G. E., \& Katok, E. (2008). Learning-by-Doing in the Newsvendor Problem: A Laboratory Investigation of the Role of Experience and Feedback. Manufacturing \& Service Operations Management, 10(3), 519-538. https://doi.org/10.1287/msom.1060.0190

Chen, K. G., Song, X. F., Wang, X. Y., \& Huang, M. (2016). Joint Pricing and Production Decisions with the Overconfident Sales Agent. Journal of Systems \& Management, 25(3), 468-475.

Croson D. C., Croson, R., \& Ren, Y. (2008). How to Manage an Overconfident Newsvendor. Dallas: The University of Texas.

Gino, F., \& Pisano, G. (2008). Toward a Theory of Behavioral Operations. Manufacturing \& Service Operations Management, 10(4), 676-691. https://doi.org/10.1287/msom.1070.0205

Li, Q., \& Atkins, D. (2005). On the Effect of Demand Randomness on a Price/quantity Setting Firm. IIE Transactions, 37(12), 1143-1153. https://doi.org/10.1080/07408170500288182 
Liu, Y. S., Zhang, J. H., \& Wang, L. L. (2013). Optimal Joint Pricing and Ordering Decisions in Newsvendor Model with Two Demand Cases. Control and Decision, 28(9), 1419-1422.

Loch, C. H., \& Wu, Y. (2007). Behavioral Operations Management, Foundations and Trends® in Technology. Information and Operations Management, 1(3), 121-232. http://dx.doi.org/10.1561/0200000009

Moore, D. A., \& Healy, P. J. (2008). The Trouble with Overconfidence. Psychological Review, 115(2), 502-517. https://doi.org/10.1037/0033-295x.115.2.502

ÖZER Ö. \& ZHENG Y. (2011). Behavioral Issues in Pricing Management. In The Oxford Handbook of Pricing Management. UK: Oxford University Press.

Petruzzi, N., \& Dada, M. (1999). Pricing and the Newsvendor Problem: A Review with Extensions. Operations Research, 47, 183-194. https://doi.org/10.1287/opre.47.2.183

Ren, Y., \& Croson, R. (2013). Overconfidence in Newsvendor Orders: An Experimental Study. Management Science, 59(11), 2502-2517. https://doi.org/10.1287/mnsc.2013.1715

Schweitzer M. E., \& Cachon, G. P. (2000). Decision Bias in the Newsvendor Problem with a Known Demand Distribution: Experimental Evidence. Management Science, 46(3), 404-420. https://doi.org/10.1287/mnsc.46.3.404.12070

Tversky, A., \& Kahneman, D. (1974). Judgment under Uncertainty: Heuristics and Biases. Science, 185(4157), 1124-1131. https://doi.org/10.1017/cbo9780511809477.002

Whitin T. M. (1955). Inventory Control and Price Theory. Management Science, 2, 61-80. https://doi.org/10.1287/mnsc.2.1.61

Zhang, J. L., Zhang, X. S., \& Wang, Y. Q. (2008). Pricing and Inventory Control in Newsboy's Model with General Demand Function. Systems Engineering-Theory \& Practice, 9, $20-28$.

Zhang, P., Zhang, J., \& Ma, J. (2015). Joint Decision-making of Order Quantities and Pricing for Loss-averse Retailers with Two Demand Cases. Control and Decision, 30(10), 1820-1827. https://doi.org/10.13195/j.kzyjc.2014.1288

Zhang, Z. C. (2016). Research on Ordering and Pricing Decisions of Overconfident Retailer. Tianjin University of Technology.

\section{Copyrights}

Copyright for this article is retained by the author(s), with first publication rights granted to the journal.

This is an open-access article distributed under the terms and conditions of the Creative Commons Attribution license (http://creativecommons.org/licenses/by/4.0/). 